\title{
ERROR ANALYSIS OF AN ALGORITHM FOR SUMMING GERTAIN FINITE SERIES
}

\author{
DAVID ELLIOTT
}

(Received 18 August 1966, revised 12 February 1967)

\section{Introduction}

An algorithm for summing the series $P_{N}=\sum_{n=0}^{N} a_{n} p_{n}$, where the coefficients $a_{n}$ are assumed known, and the quantities $p_{n}$ satisfy a linear three term recurrence relation, has been given by Clenshaw [1]. If we suppose that the $p_{n}$ satisfy the recurrence relation

$$
p_{n}+\alpha_{n} p_{n-1}+\beta_{n} p_{n-2}=0,
$$

where $\alpha_{n}$ and $\beta_{n}$ are, in general, functions of $n$, then $P_{N}$ may be found by constructing a sequence $\left\{b_{n}\right\}$ for $n=N(-1) 0$, where the $b_{n}$ satisfy the inhomogeneous recurrence relation

$$
b_{n}+\alpha_{n+1} b_{n+1}+\beta_{n+2} b_{n+2}=a_{n},
$$

with the conditions,

$$
b_{N+1}=b_{N+2}=0 .
$$

The sum $P_{N}$ is then given by

$$
P_{N}=p_{0} b_{0}+\left(p_{1}+\alpha_{1} p_{0}\right) b_{1} .
$$

This result can be readily verified by multiplying each side of equation (1.2) by $p_{n}$, summing from $n=0$ to $N$, and making use of equations (1.1) and (1.3).

This algorithm is very convenient for use on a digital computer since it is easy to program, and may in fact be looked upon as a generalisation of the well known "nested-multiplication" process for summing power series. It has been frequently used for summing Chebyshev series. In this paper, we propose to discuss this algorithm in far more detail than appears to have been given previously in the literature. In Section 3 we shall give a fairly complete error analysis. Partial error analyses have already been given in particular cases. Clenshaw [1] has given a partial analysis for a series of shifted Chebyshev polynomials; Elliott [2] has generalised this result to series of ultraspherical polynomials, and Smith [3] has further extended 
this result to the case when the $p_{n}$ are general orthogonal polynomials. However, all these analyses are incomplete since they consider only the effect of the error in each $a_{n}$, and neglect the errors which might arise in computing the $b_{n}$ from equation (1.2), and $P_{N}$ from equation (1.4). In the next section we shall first discuss the algorithm in some detail.

\section{Further discussion of the algorithm}

Before proceeding with the error analysis, we shall first obtain an explicit expression for the quantities $b_{n}$, as defined by equations (1.2) and (1.3), in terms of functions which we shall consider to be known. It is well known that equation (1.1) possesses two linearly independent solutions. One of these solutions is the given $p_{n}$; let a second solution of the recurrence relation be denoted by $q_{n}$. Since these two solutions are linearly independent, we define the Casorati determinant $W_{n}$ (see, for example, Milne-Thomson [4]) by

$$
W_{n}=p_{n-1} q_{n}-p_{n} q_{n-1} .
$$

We have $W_{n} \neq 0$, and furthermore

$$
W_{n}=\beta_{n} W_{n-1} \quad \text { for } n=1,2,3, \cdots .
$$

An explicit solution for the quantities $b_{n}$ can now be given in terms of $a_{n}$, $p_{n}$ and $q_{n}$. That this is not a completely trivial problem may be seen from the fact that the homogeneous equation

$$
b_{n}+\alpha_{n+1} b_{n+\mathbf{1}}+\beta_{n+2} b_{n+2}=0
$$

is not, in general, the same as equation (1.1) satisfied by $p_{n}$ and $q_{n}$. However it can be verified by substitution that two linearly independent solutions of equation (2.3) are given by

$$
\frac{p_{n-1}}{\prod_{i=1}^{n} \beta_{i}} \text { and } \frac{q_{n-1}}{\prod_{i=1}^{n} \beta_{i}},
$$

where we have assumed (as in the cases to be considered later) that none of the $\beta_{i}$ is zero. With this result, it is then readily shown by standard techniques that

$$
b_{n}=\frac{1}{W_{n}} \sum_{m=n}^{N}\left(p_{n-1} q_{m}-p_{m} q_{n-1}\right) a_{m}, \quad \text { for } n=0(1) N .
$$

It is now convenient to introduce the sum $Q_{N}$, which is defined by

$$
Q_{N}=\sum_{n=0}^{N} a_{n} q_{n}
$$


We note that if our problem had been one of evaluating $Q_{N}$ instead of $P_{N}$, then we would have calculated precisely the same quantities $b_{n}$. The value of $Q_{N}$ is given by

$$
Q_{N}=q_{0} b_{0}+\left(q_{1}+\alpha_{1} q_{0}\right) b_{1},
$$

and of course depends on the specific values taken by $q_{0}$ and $q_{1}$, which then uniquely define all the quantities $q_{n}$. Thus we see that the quantities $b_{0}$ and $b_{1}$, may be expressed in terms of $P_{N}$ and $Q_{N}$; in fact,

$$
\left\{\begin{array}{l}
b_{0}=\frac{1}{W_{1}}\left\{\left(q_{1}+\alpha_{1} q_{0}\right) P_{N}-\left(p_{1}+\alpha_{1} p_{0}\right) Q_{N}\right\}, \\
b_{1}=-\frac{1}{W_{1}}\left\{q_{0} P_{N}-p_{0} Q_{N}\right\} .
\end{array}\right.
$$

This result is of interest in so far as we can see the conditions under which the algorithm should not be used. Suppose that $\left|q_{n}\right| \gg\left|\dot{p}_{n}\right|$ for most values of $n$ in $0(1) N$, then in general we shall have $\left|Q_{N}\right| \gg\left|P_{N}\right|$. The sum $P_{N}$ will then be obtained as the difference of two large numbers and a considerable loss of accuracy will occur.

An excellent example of such behaviour is given if we use the algorithm to sum the series

$$
P_{12}=J_{0}(1)+2 \sum_{n=1}^{6} J_{2 n}(1),
$$

where $J_{n}(x)$ denotes the Bessel function of the first kind. The value of $P_{12}$ is 1 , correct to 10 decimal places. Suppose we calculate the quantities $b_{n}$ using floating point decimal arithmetic to ten significant figures, using only single precision operations. Now we have

with

$$
b_{n}=a_{n}+2 n b_{n+1}-b_{n+2},
$$

and

$$
b_{13}=b_{14}=0
$$

$$
a_{n}= \begin{cases}2 & (n \text { even, } \neq 0), \\ 1 & (n=0), \\ 0 & (n \text { odd }) .\end{cases}
$$

The quantities $b_{n}$ for $n=4(1) 12$ may be evaluated exactly (being integers with 10 or less digits), and rounded values have to be taken for the remaining $b_{n}$. We find in particular that $b_{\mathbf{4}}=0.3279706418 \times 10^{10}$ (exact), together with $b_{0}=-0.7377245906 \times 10^{11}$ and $b_{1}=0 \cdot 1282818767 \times 10^{12}$. If now, in equation (1.4) we use values of $J_{0}(1)$ and $J_{1}(1)$ correctly rounded to ten decimal places, we find that $P_{\mathbf{1 2}}=-30$ instead of 1 , i.e. we have no correct significant digits. It is of course well known that two linearly in- 
dependent solutions of the recurrence relation satisfied by $J_{n}(1)$ are $J_{n}(1)$ and $Y_{n}(1)$ (the Bessel function of the second kind), and that for $n \gg 1$, we have $J_{n}(1) \sim 1 / 2^{n} n !$ and $Y_{n} \sim-2^{n}(n-1) ! / \pi$. This is an example where $\left|q_{n}\right| \gg\left|p_{n}\right|$ for most values of $n$ in the range $n=0(1) 12$, over which the series is summed.

The question then arises as to how such series might be summed. It is suggested that we first compute the values of $p_{n}$ from equation (1.1) by making use of Miller's recurrence algorithm. This algorithm, and the errors which arise in its use, have recently been discussed in some detail by Olver [5]. With the values of $p_{n}$ then determined, the series may be summed directly by accumulating the products $a_{n} p_{n}$ for $n=0(1) N$. We shall not discuss the summation of such series any further in this paper. In the next section we shall consider a complete error analysis of the algorithm of Section 1 .

\section{Analysis of errors}

We shall now consider the total effect of round-off errors on the computed value of $P_{N}$, due to the possible round-off errors arising at each step of the algorithm. The possible sources of error in $P_{N}$ are:

(i) the round-off error in each $a_{n}$,

(ii) errors in the computed values of $\alpha_{n}$ and $\beta_{n}$,

(iii) errors in computing the $b_{n}$ from equation (1.2), and

(iv) errors in computing $P_{N}$ from equation (1.4).

Previous error analyses [1], [2] and [3] have only considered the error in $P_{N}$ due to the errors in the $a_{n}$. Smith [3] correctly noted that if there is an error $\phi_{n}$ in each $a_{n}$ then the error in $P_{N}$, assuming that no other errors occur, is obviously $\sum_{n=0}^{N} \phi_{n} p_{n}$. Clenshaw [1] and Elliott [2] obtained this result in their particular cases, in a far more elaborate manner.

In the subsequent analysis, we shall denote by $\bar{u}$ the value of a quantity $u$ as it is computed during the course of the algorithm. We note that $\bar{u}$ is not necessarily always the correctly rounded value of $u$. The calculations may be performed in either fixed or floating point arithmetic, and to any number base. In particular if we assume that we are working in fixed point arithmetic to $t$ decimal places, then $\bar{u}$ is the correctly rounded value of $u$ if $|u-\bar{u}| \leqq \frac{1}{2} \times 10^{-t}$. On the other hand, if we are working with floating point numbers, with $\bar{u}=a \cdot 10^{b}$ where $0 \cdot 1 \leqq|a|<1, b$ an integer and $a$ given to $t$ decimal places, then $\bar{u}$ is the correctly rounded value of $u$ if $|u-\bar{u}| \leqq \frac{1}{2}|u| \times 10^{1-t}$. In both cases we can put an upper bound on the round-off error; the modification for binary arithmetic is straightforward.

Let us now consider the algorithm again, but in terms of quantities 
that are actually computed. First, from equation (1.2), we compute $\bar{b}_{n}$ say, where

$$
\bar{b}_{n}=\left(\bar{a}_{n}-\bar{\alpha}_{n+1} \bar{b}_{n+1}-\bar{\beta}_{n+2} \bar{b}_{n+2}\right)+r_{n} .
$$

The quantity $r_{n}$ is introduced as the round-off error which arises on rounding off the quantity in the brackets $($ ). It will depend upon the way in which the computation of $\left(\bar{a}_{n}-\bar{\alpha}_{n+1} \bar{b}_{n+1}-\bar{\beta}_{n+2} \bar{b}_{n+2}\right)$ is performed, and will, for example, be less if the sum of products is accumulated to double, rather than single length. Since equation (3.1) is not suitable for analysis as it stands, we rewrite it as

$$
\bar{b}_{n}=\bar{a}_{n}-\alpha_{n+1} \bar{b}_{n+1}-\beta_{n+2} \bar{b}_{n+2}+\varepsilon_{n}+r_{n},
$$

where the quantity $\varepsilon_{n}$ is defined by

$$
\varepsilon_{n}=\left(\alpha_{n+1}-\bar{\alpha}_{n+1}\right) \bar{b}_{n+1}+\left(\beta_{n+2}-\bar{\beta}_{n+2}\right) \bar{b}_{n+2} \text {. }
$$

Now we may write,

$$
\varepsilon_{n}=\left(\alpha_{n+1}-\bar{\alpha}_{n+1}\right) b_{n+1}+\left(\beta_{n+2}-\bar{\beta}_{n+2}\right) b_{n+2},
$$

approximately, on neglecting second order small quantities such as $\left(\alpha_{n+1}-\bar{\alpha}_{n+1}\right)\left(b_{n+1}-\bar{b}_{n+1}\right)$ etc.

Let $\phi_{n}$ and $\psi_{n}$ denote the errors in $a_{n}$ and $b_{n}$ respectively, i.e. we define

$$
\left\{\begin{array}{l}
\bar{a}_{n}=a_{n}+\phi_{n} \text { and } \bar{b}_{n}=b_{n}+\psi_{n} \text { for } n=0(1) N \\
\text { where } \phi_{n}=\psi_{n}=0 \text { for } n=N+1, N+2 .
\end{array}\right.
$$

On subtracting equation (1.2) from (3.2), we find that the errors $\psi_{n}$ are completely defined by

$$
\left\{\begin{array}{l}
\psi_{n}+\alpha_{n+1} \psi_{n+1}+\beta_{n+2} \psi_{n+2}=\phi_{n}+\varepsilon_{n}+r_{n}, \\
\text { where } \psi_{N+1}=\psi_{N+2}=0 .
\end{array}\right.
$$

Thus the quantities $\psi_{n}$ satisfy a set of equations similar to that of the $b_{n}$, although with different right hand sides. On comparing with equation (1.4), we have immediately that

$$
p_{0} \psi_{0}+\left(p_{1}+\alpha_{1} p_{0}\right) \psi_{1}=\sum_{m=0}^{N}\left(\phi_{m}+\varepsilon_{m}+r_{m}\right) p_{m} .
$$

We shall make use of this result when we consider the errors in the evaluation of the sum $P_{N}$.

Finally, from equation (1.4), we actually compute a quantity $\bar{P}_{N}$ say, where

$$
\bar{P}_{N}=\left[\bar{p}_{0} \bar{b}_{0}+\left(\bar{p}_{1}+\bar{\alpha}_{1} \bar{p}_{0}\right) \bar{b}_{1}\right]+s,
$$

where the quantity $s$ performs a similar role to that of $r_{n}$ in equation (3.2), 
i.e., it is the round-off error introduced on evaluating the sum of products in the brackets [ ]. If we rewrite $\bar{P}_{N}$ as

$$
\bar{P}_{N}=p_{0} \delta_{0}+\left(p_{1}+\alpha_{1} p_{0}\right) \delta_{1}+\xi+s,
$$

where $\xi$ is defined by

$$
\xi=\left(\bar{p}_{0}-p_{0}\right) \bar{b}_{0}+\left(\bar{p}_{1}-p_{1}\right) \bar{b}_{1}+\left[\bar{\alpha}_{1}\left(\bar{p}_{0}-p_{0}\right)+\left(\bar{\alpha}_{1}-\alpha_{1}\right) p_{0}\right] \bar{b}_{1},
$$

then on subtracting equation (1.4) from (3.9), we have

$$
\bar{P}_{N}-P_{N}=p_{0} \psi_{0}+\left(p_{1}+\alpha_{1} p_{0}\right) \psi_{1}+\xi+s
$$

If we now make use of equation (3.7), we have the required result,

$$
\bar{P}_{N}-P_{N}=\sum_{m=0}^{N}\left(\phi_{m}+\varepsilon_{m}+r_{m}\right) p_{m}+\xi+s
$$

Previous analyses ([1], [2] and [3]) have essentially given only the partial result that $\bar{P}_{N}-P_{N}=\sum_{m=0}^{N} \phi_{m} p_{m}$, and this is not necessarily the major contribution to the total error.

Our previous example on summing a series of Bessel functions is a good example of this. Here, the coefficients $a_{m}$ are given exactly so that $\phi_{m}=0$. Furthermore, $\varepsilon_{m}=0$ since the coefficients $\alpha_{m}$ and $\beta_{m}$ are also given exactly. Thus the error $\bar{P}_{12}-P_{12}$ is given by

$$
\bar{P}_{12}-P_{12}=\sum_{m=0}^{12} r_{m} p_{m}+\xi+s
$$

in this example, and as we have already seen this is not negligible with respect to $P_{\mathbf{1 2}}$.

The results derived in this and the previous section provide sufficient information for an estimate of the error in $P_{N}$ to be made. In the next section we propose to give a complete analysis of the error which may arise when we sum a finite series of Chebyshev polynomials.

\section{Errors in summing a Chebyshev series}

One frequently needs to evaluate a sum of the form

$$
P_{N}=\sum_{n=0}^{N} a_{n} T_{n}(x), \text { for }-1 \leqq x \leqq 1,
$$

where $T_{n}(x)$ is the Chebyshev polynomial of the first kind defined by

$$
T_{n}(x)=\cos n \theta \text { where } x=\cos \theta \text {, for } n=0,1,2, \cdots \text {. }
$$

These polynomials satisfy the recurrence relation

$$
T_{n}(x)-2 x T_{n-1}(x)+T_{n-2}(x)=0,
$$


a second solution of which are the Chebyshev polynomials of the second kind, $U_{n}(x)$ which are defined by

$$
U_{n}(x)=\frac{\sin (n+1) \theta}{\sin \theta}, x=\cos \theta, n=0,1,2, \cdots
$$

It can be readily shown that, in the notation of Section 2 ,

$$
\frac{p_{n-1} q_{m}-p_{m} q_{n-1}}{W_{n}}=U_{m-n}(x),
$$

so that from equation (2.4), we have

$$
b_{n}=\sum_{m=n}^{N} U_{m-n}(x) a_{m} .
$$

In order to carry out the error analysis, we shall assume that the calculation is done in fixed point arithmetic to $t$ decimal places. We shall further assume that $\left|r_{n}\right|,|s| \leqq \frac{1}{2} \times 10^{-t}$. Since, in general, the quantity $x$ will not be represented exactly, let us assume that in the calculations it is replaced by $\bar{x}$, where $\bar{x}-x=\gamma$, say. Then since $\alpha_{n}=-2 x$ we have $\alpha_{n}-\bar{\alpha}_{n}=2 \gamma$ for all $n$. Finally since $\beta_{n}=1$, for all $n$, we shall assume that $\beta_{n}-\bar{\beta}_{n}=0$. Equation (3.4) now gives

$$
\varepsilon_{n}=2 \gamma b_{n+1} \text {, }
$$

approximately. Since $p_{0}=1$ and $p_{1}=x$ we shall assume that $\vec{p}_{0}-p_{0}=0$ and $\bar{p}_{1}-p_{1}=\gamma$. Thus, from equation $(3.10)$ we have

$$
\xi=-\gamma b_{1},
$$

approximately, where we have replaced $b_{1}$ by $b_{1}$. With these results, equation (3.12) gives

$$
\bar{P}_{N}-P_{N}=\sum_{m=0}^{N}\left(\phi_{m}+r_{m}\right) T_{m}(x)+2 \gamma \sum_{m=0}^{N} b_{m+1} T_{m}(x)-\gamma b_{1}+s .
$$

Now the second sum on the right hand side of this equation may be summed explicitly, for

$$
\begin{aligned}
\sum_{m=0}^{N} b_{m+1} T_{m}(x) & =\sum_{m=1}^{N} b_{m} T_{m-1}(x), \text { since } b_{N+1}=0 \\
& =\sum_{m=1}^{N}\left(\sum_{k=m}^{N} U_{k-m}(x) a_{k}\right) T_{m-1}(x), \text { by equation }(4.6) \\
& =\sum_{k=1}^{N} a_{k}\left(\sum_{m=1}^{k} U_{k-m}(x) T_{m-1}(x)\right) \\
& =\sum_{k=1}^{N} a_{k}\left(\frac{k+1}{2}\right) U_{k-1}(x) .
\end{aligned}
$$


Thus equation (4.9) may be rewritten as

$$
\bar{P}_{N}-P_{N}=\sum_{m=0}^{N}\left(\phi_{m}+r_{m}\right) T_{m}(x)+\gamma \sum_{m=1}^{N} m a_{m} U_{m-1}(x)+s .
$$

At this point, we may obtain an upper bound for $\left|\bar{P}_{N}-P_{N}\right|$ by making use of the facts that for $-1 \leqq x \leqq 1,\left|T_{m}(x)\right| \leqq 1$ and $\left|U_{m-1}(x)\right| \leqq m$. If we further assume that both $\left|\phi_{m}\right|$ and $|\gamma|$ are less than $\frac{1}{2} \times 10^{-t}$, we have

$$
\left|\bar{P}_{N}-P_{N}\right| \leqq \frac{1}{2} \times 10^{-t}\left\{(2 N+3)+\sum_{m=1}^{N} m^{2}\left|a_{m}\right|\right\} .
$$

At this point, we may recall Clenshaw's partial error analysis [1], which although given in terms of shifted Chebyshev polynomials would give for this problem $\left|\bar{P}_{N}-P_{N}\right| \leqq \frac{1}{2} \times 10^{-t} \times(2 N+2)$. (Clenshaw introduced a factor of 2 to account for possible round-off errors in $b_{n}$ ). The additional term given in equation (4.11) is only likely to make a significant contribution to the error if we are summing a Chebyshev series where the coefficients $a_{n}$ are slowly convergent. In numerical work a "slowly convergent" series would be one for which $a_{n}=0\left(1 / n^{2}\right)$. If we assume that there exists a constant $A$ such that $\left|a_{n}\right| \leqq A / n^{2}$ for all $n \geqq 1$, then equation (4.11) gives the result that

$$
\left|\bar{P}_{N}-P_{N}\right| \leqq \frac{1}{2} \times 10^{-t}\{(2+A) N+3\} \text {. }
$$

\section{Conclusion}

In this paper we have given a fairly complete discussion of the algorithm which may be used to sum a series $\sum_{n=0}^{N} a_{n} p_{n}$ where the $p_{n}$ satisfy a three term recurrence relation. We have seen that the algorithm should not be used for series in which the second solution of the recurrence relation is considerably larger in modulus than $\left|p_{n}\right|$. For problems where the algorithm may be used, a complete discussion of the errors has been given, from which a bound may be obtained for the errors in the final sum.

\section{Acknowledgement}

This research has been sponsored in part by the United States Air Force Office of Scientific Research under Contract AF-AFOSR-660-64. The author wishes to thank Messrs. J. D. Donaldson and M. Rush for their comments. 


\section{References}

[1] C. W. Clenshaw, 'A note on the summation of Chebyshev series', M.T.A.C. 9 (1955), $118-120$.

[2] D. Elliott, 'On the expansion of functions in ultraspherical polynomials', Journ. Aust. Math. Soc. 1 (1960), 428-438.

[3] F. J. Smith, 'An algorithm for summing orthogonal polynomial series and their derivatives with applications to curve-fitting and interpolation', Math. Comp. 19 (1965), 33-36.

[4] L. M. Milne-Thomson, The calculus of finite differences, (Macmillan, London, 1933).

[5] F. W. J. Olver, 'Error analysis of Miller's recurrence algorithm', Math. Comp. 18 (1964), 65-74.

Mathematics Department

University of Tasmania

Hobart, Tasmania 